\title{
Restoration of miR-26b expression partially reverses the cisplatin resistance of NSCLC by targeting tafazzin
}

This article was published in the following Dove Press journal: OncoTargets and Therapy

\section{Shuzhi Zang' \\ Shasha Zhao ${ }^{2}$ \\ Xinyuan Gao' \\ Yunxia $\mathrm{Li}^{3}$ \\ Chunlei Zhong ${ }^{3}$ \\ Jianlian $\mathrm{Gao}^{4}$}

'Respiratory Ward I, The First Affiliated Hospital of Xinxiang Medical University, Weihui, Henan 453100, People's Republic of China; ${ }^{2}$ Respiratory Intensive Care Unit, The First Affiliated Hospital of Xinxiang Medical University, Weihui, Henan 453100, People's Republic of China; ${ }^{3}$ Respiratory Ward 2, The First Affiliated Hospital of Xinxiang Medical University, Weihui, Henan 453100, People's Republic of China; ${ }^{4}$ Clinical Pharmacy, The First Affiliated Hospital of Xinxiang Medical University, Weihui, Henan 453100, People's Republic of China
Correspondence: Shuzhi Zang Respiratory Ward I, The First Affiliated Hospital of Xinxiang Medical University, No. 88, Jiankang Road, Weihui City, Henan Province 453100, People's Republic of China

Email zangshuzhi0I@I26.com
Background: Dysregulation of microRNAs has been reported to be responsible for drug resistance of cancers. However, the association between aberrant expression of miR-26b and cisplatin resistance in non-small cell lung cancer (NSCLC) remains unclear.

Methods: PC9 and A549 were used to establish the cisplatin resistance models on NSCLC. Expression of miR-26b in cisplatin-resistant PC9 and A549 cells (PC9/R and A549/R) was detected by quantitative real-time PCR assays. Drug sensitivity and mitochondrial apoptosis were detected by Cell Counting Kit- 8 assay and flow cytometry assay, respectively. The target relationship between miR-26b and tafazzin (TAZ) was validated by dual-luciferase reporter assay.

Results: Obvious downregulation of miR-26b was observed in PC9/R and A549/R cells. Restoration of miR-26b partially reversed the cisplatin resistance of PC9/R and A549/R cells. Expression of TAZ was increased in PC9/R and A549/R cells compared to the parental PC9 and A549 cells. Results of dual-luciferase reporter assays verified that TAZ was targeted by miR-26b. We showed that restoration of miR-26b expression inhibited the TAZ expression and thus expanded the mitochondrial pathway of apoptosis induced by cisplatin in PC9/ $\mathrm{R}$ and A549/R cells.

Conclusion: Restoration of miR-26b expression partially reverses the cisplatin resistance of NSCLC by targeting TAZ. miR-26b/TAZ axis may represent a potential strategy to reverse the cisplatin in NSCLC.

Keywords: NSCLC, cisplatin, resistance, miR-26b, TAZ

\section{Introduction}

Non-small cell lung cancer (NSCLC) is the most common malignant tumor with poor prognosis in the world. Despite surgery is the most effective treatment for NSCLC, a majority of the NSCLC cases are diagnosed at the advanced stages. Furthermore, because of the high invasion and early metastasis of NSCLC, the 5-year survival rate of NSCLC is very low. ${ }^{1,2}$ For patients with advanced NSCLC, systemic chemotherapy is an important strategy. 3,4

Among the systemic chemotherapeutic drugs, platinum-based agents are first-line drugs that used in the treatment of NSCLC." ${ }^{5,6}$ Among the platinum-based drugs, cisplatin is the most commonly used drug in the clinics. Cellular cisplatin can crosslink with cellular DNA and thus induce apoptosis of NSCLC cells. ${ }^{7,8}$ However, drug resistance usually occurs during the chemotherapy treatment course. ${ }^{9,10}$ To solve this problem, some novel strategies are required to be explored to reverse or impede the cisplatin resistance in NSCLC treatment. 
MicroRNAs (miRNAs) are endogenous, small and noncoding RNA molecules that target approximately $60 \%$ of human genes. ${ }^{11,12}$ MiRNAs can bind to $3^{\prime}$-untranslated regions ( $3^{\prime}$-UTRs) of their targeted mRNAs and thus decrease the stability of them. As miRNAs suppress the expression of their targeted genes, they participate in various biological processes including cell proliferation, differentiation and apoptosis. Dysregulation of miRNAs has been proved to promote tumorigenesis and cancer progression. ${ }^{13-15}$ Furthermore, previous studies indicate that abnormal expression of miRNAs promotes drug resistance in cancers. ${ }^{16-18}$ Thus, the expression profile of miRNA is an important factor for influencing the cisplatin sensitivity of NSCLC cells. In the present study, we showed significant downregulation of miR$26 \mathrm{~b}$ in cisplatin-resistant NSCLC cells. Thus, we focused on exploring the association of miR-26b expression and cisplatin resistance in NSCLC.

\section{Materials and methods}

\section{Cell culture}

Human NSCLC cell lines PC9 and A549 were obtained from the China Center for Type Culture Collection (CCTCC, Wuhan, China) and cultured in Roswell Park Memorial Institute-1640 medium (RPMI-1640; Invitrogen, Carlsbad, CA, USA). All of the cells were supplemented with $10 \%$ FBS and maintained in a humidified incubator at $37^{\circ} \mathrm{C}$. For the acquisition of cisplatin-resistant NSCLC cell lines, PC9 and A549 cells were gradually exposed to increasing concentrations of cisplatin from 0.2 to $1 \mu \mathrm{M}$. Briefly, PC9 and A549 cells were initially treated with $0.2 \mu \mathrm{M}$ cisplatin for 8 weeks. Subsequently, cisplatin concentration was increased every 3 weeks by $0.1 \mu \mathrm{M}$ up to a final concentration of $1 \mu \mathrm{M}$. The acquired cisplatin-resistant PC9 and A549 were named as PC9/R and A549/R, respectively. Before experiments on PC9/R and A549/R cells, they were moved to the cisplatinfree culture medium for 2 weeks to eliminate the interference of residual cisplatin.

\section{Quantitative real-time PCR}

Total RNAs from cell lines were extracted by using TRIzol reagent (Life Technologies, Carlsbad, CA, USA). Subsequently, One Step PrimeScript miRNA cDNA Synthesis Kit (TaKaRa Bio, Inc., Otsu, Japan) was used to produce the cDNA. Real-time quantitative PCR was then carried out by using the synthesized cDNA and SYBR Premix Ex Taq (TaKaRa Bio, Inc.) on a MiniOpticon real-time PCR system (Bio-Rad, Hercules,
CA, USA). The relative expression of miR-26b was measured by using the U6 small nuclear RNA (snRNA) as the internal reference.

\section{Cell transfection}

Hsa-miR-26b mimics, hsa-miR-26b antisense oligonucleotides (anti-miR-26b), negative control oligonucleotide (NCO) and Tafazzin (TAZ) short interfering RNA (siRNA) were purchased from GenePharma (Shanghai, China). pcDNA3.1 plasmid was also obtained from GenePharma. The TAZ plasmid was constructed by inserting the open reading frame of TAZ gene into the pcDNA3.1 vector. For transfection, $50 \mathrm{pmol} / \mathrm{mL}$ of various RNA oligonucleotides and $2 \mu \mathrm{g} / \mathrm{mL}$ TAZ plasmid were transfected into cells by using Lipofectamine ${ }^{\mathrm{TM}}$ 2000 (Invitrogen) according to the manufacturer's instructions.

\section{Cell viability assay}

After $24 \mathrm{hrs}$ transfection, cells were inoculated into 96well plates at a density of $1 \times 10^{4}$ cells/well with RPMI1640 culture medium. Next, the adherent cells were treated with different concentrations of cisplatin followed by incubation for $48 \mathrm{hrs}$ at $37^{\circ} \mathrm{C}$. Subsequently, cells were incubated by $10 \mu \mathrm{L}$ Cell Counting Kit-8 (CCK-8) for $2 \mathrm{hrs}$ according to the manufacturer's protocol. The absorbance was determined at $450 \mathrm{~nm}$ by using a microplate reader (Bio-Rad). Relative cell viability of experimental groups was normalized to the NCO group. 50\% inhibiting concentration (IC50) of cisplatin was calculated according to the cell viability curve.

\section{Luciferase reporter assay}

Fragment of wild type (wt) TAZ 3'-UTR containing predicted binding site paired with miR-26b was amplified and subcloned into the pGL3 Luciferase Reporter Vector (Promega, Madison, WI, USA). The recombinant pGL3 reporter was named as pGL3-wt TAZ. A site-directed mutagenesis kit (TaKaRa Bio, Inc.) and pGL3-wt TAZ were then used to create recombinant pGL3 reporter containing mutant type (mt) TAZ 3'-UTR (pGL3-mt TAZ). To perform the luciferase reporter assay, cells were co-transfected with miR-26b mimic or anti-miR-26b or NCO plus pGL3-wt TAZ or pGL3-mt TAZ by using Lipofectamine 2000; 48 hrs after transfection, luciferase activities were measured by using the Dual-Luciferase Reporter assay system (Promega, Fitchburg, WI, USA) according to the manufacturer's instructions. 


\section{Western blot analysis}

To prepare the protein samples, cells were lysed by using RIPA buffer (Cell Signaling Technologies, Danvers, MA, USA) or Mitochondria/Cytosol Fraction Kit (BioVision, Milpitas, CA, USA) to separate the cell mitochondria and cytosol according to the manufacturer's instruction. Subsequently, equal amount of protein samples were separated by $10 \%$ SDS-PAGE followed by transference to polyvinylidene difluoridemembranes (Millipore, Billerica, MA, USA) through electroblotting. The membranes were incubated overnight with primary antibodies against human TAZ, cytochrome c, caspase-9, caspase-3, GAPDH and Cox IV (Santa Cruz Biotechnology, Santa Cruz, CA, USA). After washing and removing the residual primary antibodies with PBS, membranes were probed with secondary antibodies conjugated with horseradish peroxidase. Western bolt images were detected by using Li-COR Odyssey 9120 Imaging System (LI-COR Biotechnology, Superior Street Lincoln, Nebraska, USA).

\section{Flow cytometry analysis}

Mitochondrial membrane potential (MMP) and cell apoptosis were detected by flow cytometry. To detect the MMP, cells were resuspended in PBS and stained with 5,5',6,6'tetrachloro-1,1',3,3'-tetraethyl-imidacarbo-cyanine iodide (JC-1; Molecular Probes, Waltham, MA, USA) as an indicator. Cells with high MMP emits red fluorescence and cells with low MMP emits green fluorescence. To measure the cell apoptotic rate, cells were collected and stained with FITC and propidium iodide by using an Annexin V-FITC Apoptosis Detection Kit (Sigma-Aldrich, St Louis, MO, USA) according to the manufacturer's instructions. The Annexin V-positive cells were considered as the apoptotic cells.

\section{Statistical analysis}

All data are represented as the mean \pm SD. Statistical analysis was conducted by using SPSS 16.0 software (SPSS Inc., Chicago, IL, USA) and GraphPad Prism (version 5.0; GraphPad Software, Inc., La Jolla, CA, USA). Two-tailed Student's $t$-tests were used to estimate the statistical differences between two groups. One-way ANOVA and Bonferroni's post hoc test were used to determine the differences between three or more groups. $P<0.05$ was considered statistically significant.
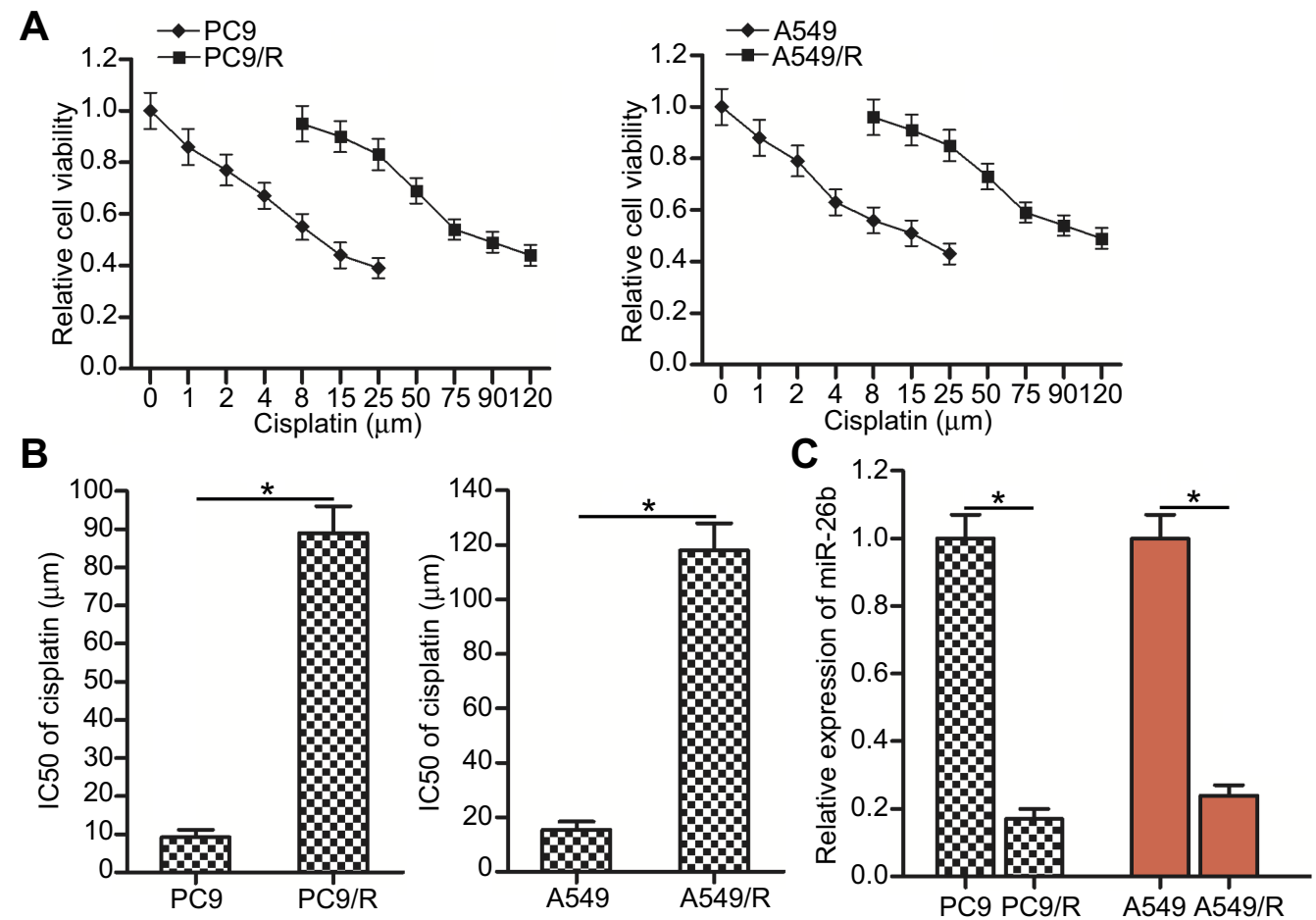

Figure I MiR-26b was downregulated in cisplatin-resistant NSCLC cells. (A) CCK-8 assays were performed to detect the drug sensitivity of A549, A549/R, PC9 and PC9/R cells to cisplatin (0-120 $\mu$ M). (B) IC50 of cisplatin to A549, A549/R, PC9 and PC9/R cells was calculated according to the cell viability curve. (C) Quantitative real-time PCR analysis was used to compare the miR-26b level between cisplatin-resistant NSCLC cells and routine NSCLC cells.

Notes: Data are expressed as mean \pm SD. $* P<0.05$.

Abbreviations: NSCLC, non-small cell lung cancer; CCK-8, Cell Counting Kit-8; IC50, 50\% inhibiting concentration. 


\section{Results}

\section{Downregulation of miR-29b in cisplatin-} resistant NSCLC cells

To study the cisplatin resistance in NSCLC, we established the cisplatin-resistant PC9 and A549 cell lines (PC9/R and A549/ $\mathrm{R})$ through continuous exposure to cisplatin. We then tested the cisplatin sensitivity of PC9/R and A549/R cells and their parental PC9 and A549 cells through CCK-8 assays. The results showed that the cell viability of PC9/R and A549/R cells was higher than the PC9 and A549 cells under the treatment of different concentrations of cisplatin (Figure 1A). According to the cell viability curve, we showed that IC50 of cisplatin to $\mathrm{PC} 9 / \mathrm{R}$ was 8.67 fold higher than its parental A549 cell line. Meanwhile, IC50 of cisplatin to A549/R was 6.68 fold higher than its parental A549 cell line (Figure 1B). We thus demonstrated that our established PC9/R and A549/R cells were resistant to cisplatin treatment. After the detection of some miRNAs through quantitative real-time PCR assays, we found that expression level of miR-29b was dramatically decreased in PC9/R and A549/R cells compared to their parental PC9 and A549 cells, respectively.

\section{Restoration of miR-26b expression partially reverses the cisplatin resistance of NSCLC}

To investigate whether downregulation of miR-26b was responsible for cisplatin resistance in PC9/R and A549/R, we restored the miR-26b expression in PC9/R and A549/R cells through transfection with miR-26b mimics (Figure 2A). We found that restoration of miR-26b
A

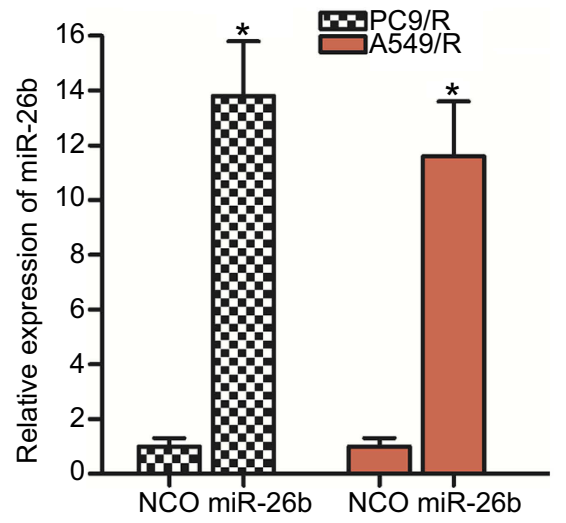

C

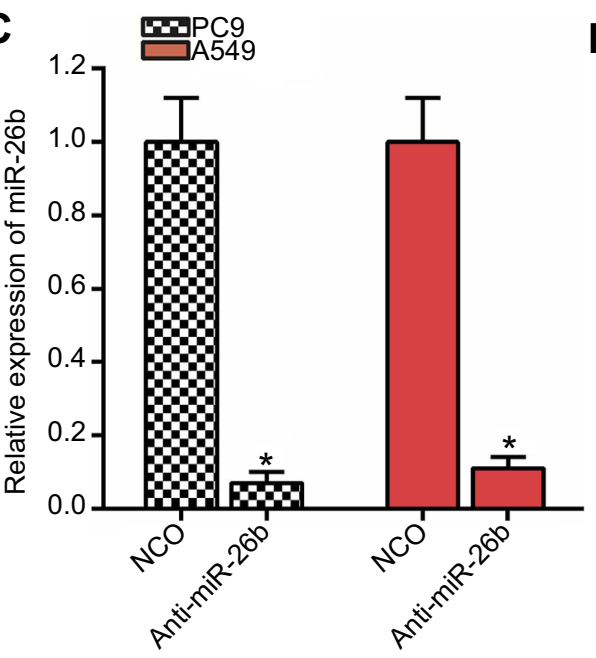

B
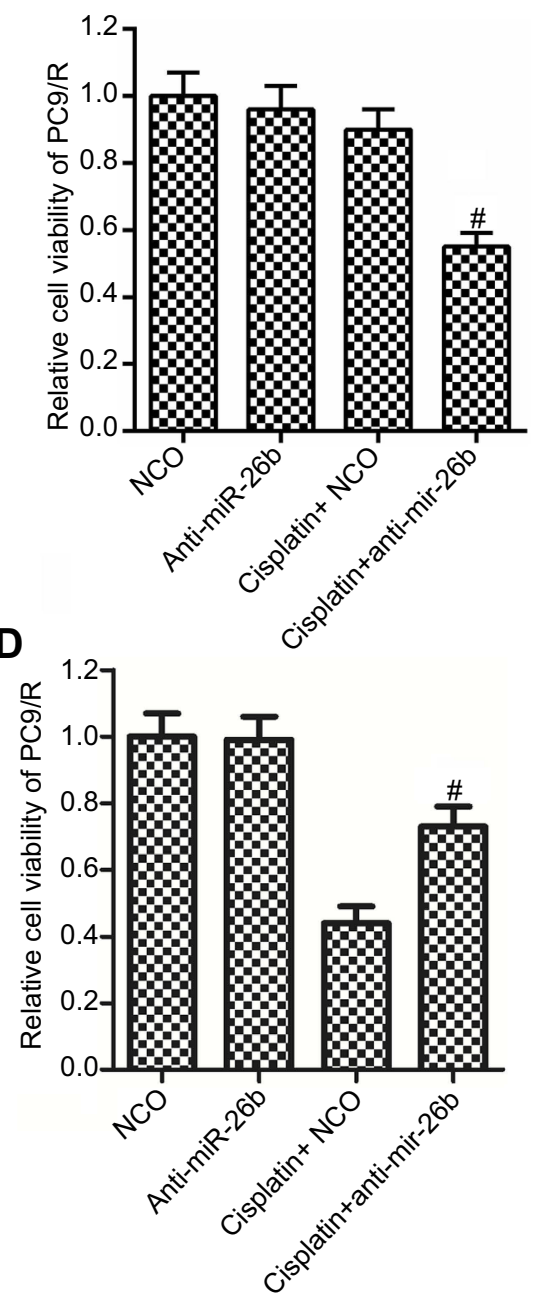
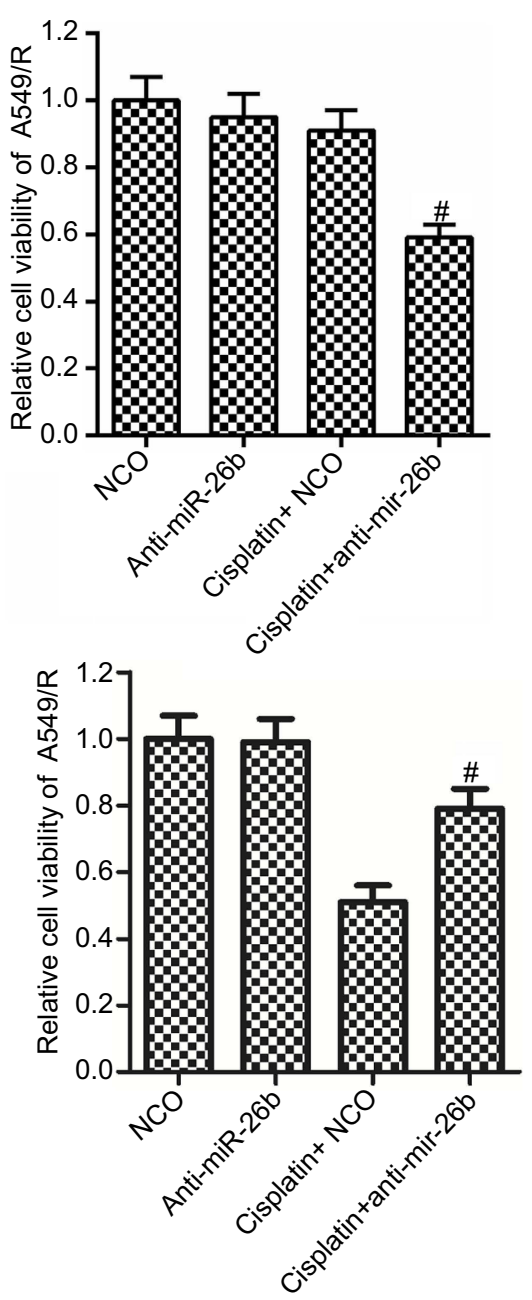

Figure 2 MiR-26b partially reversed the cisplatin resistance of PC9/R and A549/R. (A) Transfection with miR-26b mimics increased the miR-26b level in PC9/R and A549/R cells. (B) Transfection with miR-26b mimics increased the sensitivity of PC9/R and A549/R cells to cisplatin (I5 $\mu$ M). (C) Transfection with anti-miR-26b decreased the miR26b level in PC9 and A549 cells. (D) Transfection with anti-miR-26b decreased the sensitivity of PC9 and A549 cells to cisplatin (I5 $\mu$ M).

Notes: Data are expressed as mean \pm SD. $* P<0.05$ vs NCO group, ${ }^{\#} P<0.05$ vs Cisplatin+NCO group.

Abbreviation: NCO, negative control oligonucleotide. 
expression significantly increased the sensitivity of PC9/R and A549/R cells to cisplatin treatment (Figure 2B). On the other hand, we knockdown the miR-26b in routine PC9 and A549 cells by using miR-26b antisense-oligonucleotides (Figure 2C). We found that inhibition of miR-26b partially induced cisplatin resistance in these PC9 and A549 cells (Figure 2D). Taken together, we demonstrated that downregulation of miR-26b was responsible for the formation of cisplatin resistance in NSCLC. Furthermore, restoration of miR-26b expression may partially reverse the resistance of cisplatin to NSCLC cells.

\section{miR-26b targets TAZ in cisplatin-resistant NSCLC cells}

To explore the potential mechanism by which miR-26b decreased the cisplatin resistance of NSCLC cells, public miRNA databases of TargetScan, miRanda, and PicTar were used to search the potential target of miR-26b. All of these three databases showed that TAZ mRNA contained miR-26b binding sequence at the 3 '-UTR (Figure 3A). Furthermore, we observed that the expression level of TAZ was increased (Figure 3B), whereas miR-26b level was decreased (Figure 1C) in cisplatin-resistant NSCLC cells compared to their parental cells. Therefore, we inferred that miR-26b targeted TAZ in PC9/R and A549/R cells. For example in the relationship between miR-26b and TAZ, we transfected the related NSCLC cells with miR-26b or anti-miR-26b before the detection of TAZ expression through Western blot analysis. We found that transfection with miR-26b mimics reversed the overexpression of TAZ in PC9/R and A549/R cells (Figure 3C); meanwhile, knockdown of miR-26b induced TAZ overexpression in PC9 and A549 (Figure 3D). Moreover, results of luciferase reporter assays showed that miR-26b mimics decreased the luciferase activity of pGL3 luciferase reporters contained wild type TAZ (pGL3-wt TAZ), whereas anti-miR-26b increased the luciferase activity of pGL3-wt TAZ. However, either miR-26b mimics or anti-miR-26b failed to change the luciferase activity of pGL3 luciferase reporters contained mutant type TAZ (pGL3-mt TAZ) (Figure 3E). Taken together, we demonstrated that miR-26b targeted TAZ in NSCLC. Downregulation of miR-26b was responsible for the overexpression of TAZ in cisplatin-resistant NSCLC cells.
A

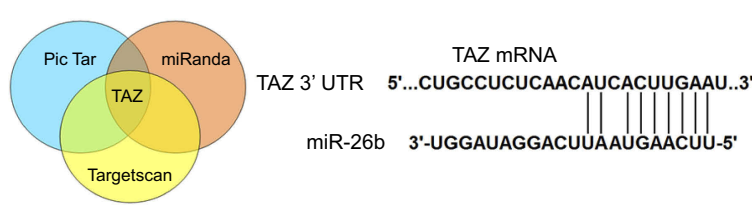

\section{E}

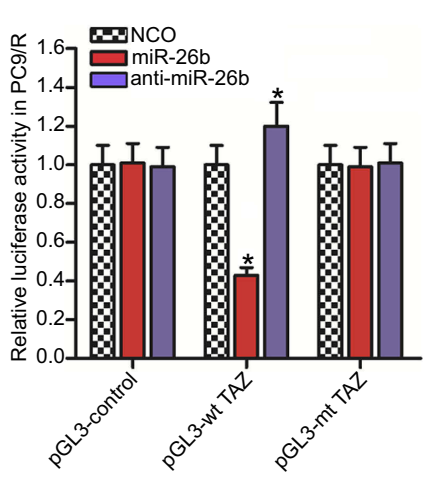

B

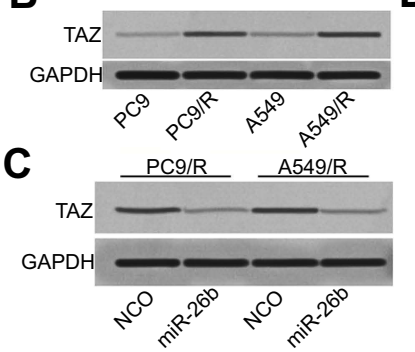

D
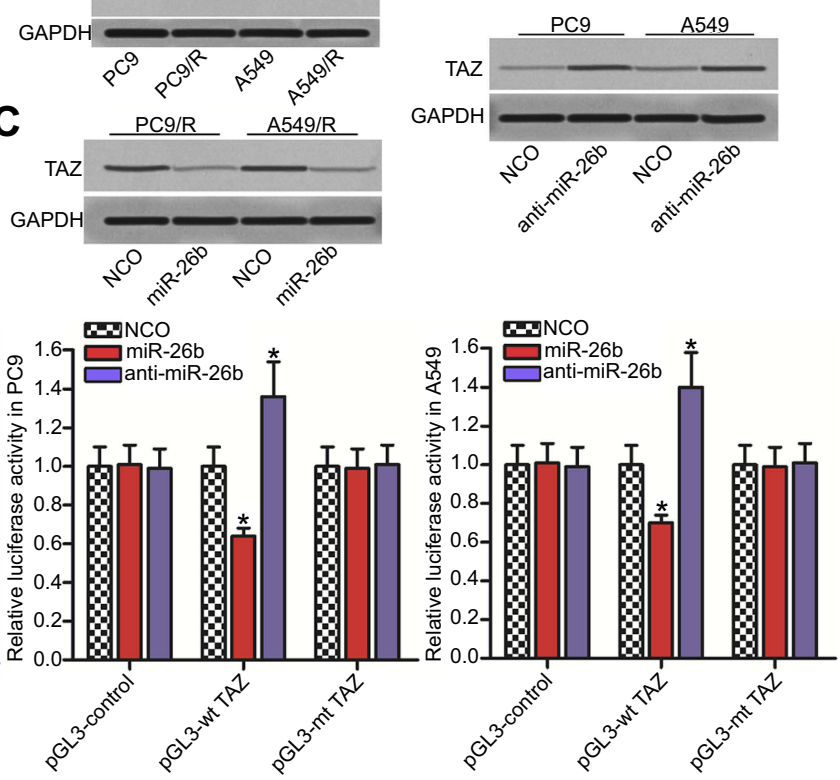

Figure 3 TAZ is the target of miR-26b in NSCLC. (A) TAZ was commonly predicted to be the target of miR-26b on TargetScan, miRanda and PicTar databases. (B) Protein level of TAZ in A549, A549/R, PC9 and PC9/R cells was evaluated by Western blot analysis. (C) Transfection with miR-26b decreased the protein level of TAZ in A549/R and PC9/R cells. (D) Transfection with anti-miR-26b increased the protein level of TAZ in A549 and PC9 cells. (E) After co-transfection with miR-26b (or anti-miR-26b) and PGL3 luciferase reporters carrying wild type TAZ 3'-UTR (or PGL3 luciferase reporters carrying mutant type TAZ 3'-UTR), luciferase activities were measured by using Dual-Luciferase Reporter Assay System according to the manufacturer's instruction.

Notes: Data are expressed as mean \pm SD. $* P<0.05$.

Abbreviations: TAZ, Tafazzin; NSCLC, non-small cell lung cancer; 3'-UTR, 3'-untranslated region; NCO, negative control oligonucleotide. 


\section{Restoration of miR-26b expression} partially reverses the cisplatin resistance of NSCLC through suppression of TAZ

As miR-26b targets TAZ in NSCLC, we next explored the effect of TAZ on the sensitization of miR-26b on cisplatin-induced cytotoxicity. We found that transfection with TAZ plasmid abolished the effect of miR-26b on reducing the cisplatin resistance of $P C 9 / R$ and A549/R (Figure 4A). Besides, we knockdown of TAZ directly by using specific siRNA. We then found that the effect of TAZ siRNA on sensitizing the cisplatin was similar to miR-26b (Figure 4B). These results indicated that the effect of miR-26b on partially reversing the cisplatin resistance was dependent on the suppression of TAZ. In addition, despite cisplatin significantly decreased the cell viability of routine PC9 and A549 cells, overexpression of TAZ by using TAZ plasmid was found to partially induce drug resistance to cisplatin (Figure 4C). It suggested that overexpression of TAZ induced by the downregulation of miR-26b was responsible for cisplatin resistance in NSCLC.

\section{Restoration of miR-26b expression} targets TAZ to increase the sensitivity of cisplatin-resistant NSCLC cells to cisplatin-induced mitochondrial

\section{apoptosis}

TAZ which is targeted by miR-26b is a mitochondriarelated protein that negatively regulates apoptosis, ${ }^{19,20}$ we next studied the role of miR-26b/TAZ axis in cisplatin-induced mitochondrial apoptosis pathway in PC9/ $\mathrm{R}$ and A549/R. Results of JC-1 staining showed that overexpression of miR-26b obviously increased the effect of cisplatin on breaking the MMP. However, transfection with TAZ plasmid reduced the collapse of mitochondria in cisplatin and miR-26b co-treated PC9/R and A549/R cells (Figure 5A). As the results of mitochondria collapse, we next found that overexpression of miR-26b obviously increased the release of cytochrome c which is a mitochondria-derived and key apoptotic inducer $^{21}$ into cytosol of PC9/R and A549/R cells. However, transfection with TAZ plasmid abolished the effect of miR-26b (Figure 5B). As the downstream of cytochrome $\mathrm{c}$ release, we observed that combination
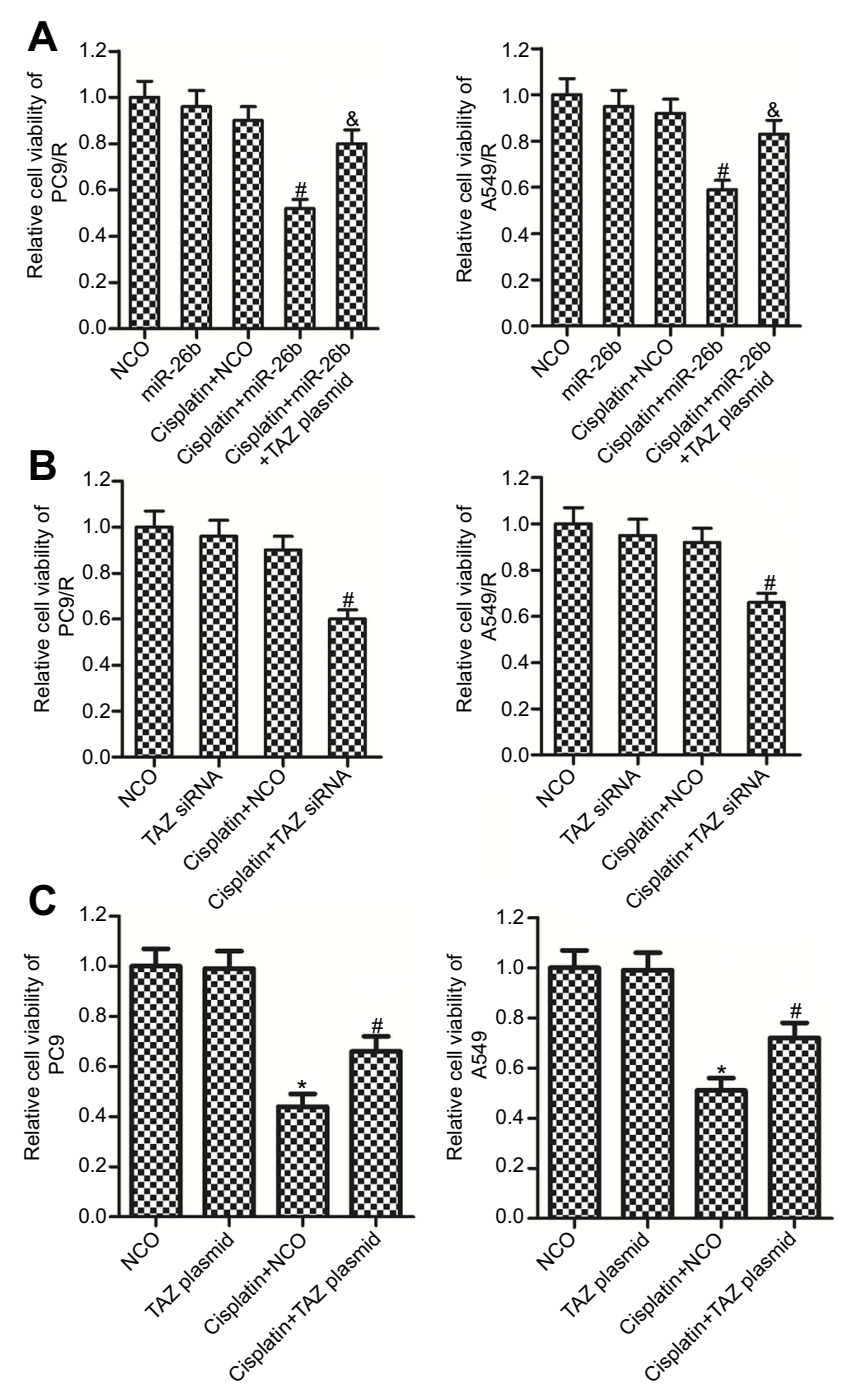

Figure 4 Restoration of miR-26b expression partially reversed the cisplatin resistance of NSCLC through suppression of TAZ. (A) Transfection with TAZ plasmid inhibited the sensitization of miR-26b on cisplatin-induced (15 $\mu \mathrm{M})$ cytotoxicity against A549/R and PC9/R. (B) Transfection with TAZ siRNA increased the sensitivity of A549/R and PC9/R cells to cisplatin (15 $\mu \mathrm{M})$ treatment. (C) Transfection with TAZ plasmid decreased the sensitivity of A549 and PC9 cells to cisplatin (I5 $\mu M)$ treatment.

Notes: Data are expressed as mean \pm SD. ${ }^{*} P<0.05$ vs NCO group, ${ }^{\#} P<0.05$ vs Cisplatin+NCO group, ${ }^{\&} P<0.05$ vs Cisplatin+miR-26b group.

Abbreviations: TAZ, Tafazzin; NSCLC, non-small cell lung cancer; NCO, negative control oligonucleotide.

with cisplatin and miR-26b induced significant cleavage of caspase-9 and caspase- 3 which are effectors of intrinsic apoptosis ${ }^{22,23}$ (Figure 5C). And finally, restoration of miR-26b expression increased the apoptotic rate of PC9/ $\mathrm{R}$ and $A 549 / \mathrm{R}$ cells which were under the cisplatin treatment through suppression of TAZ (Figure 5D). These results demonstrated that restoration of miR-26b expression targets TAZ to increase the sensitivity of cisplatin-resistant NSCLC cells to cisplatin-induced mitochondrial apoptosis. 

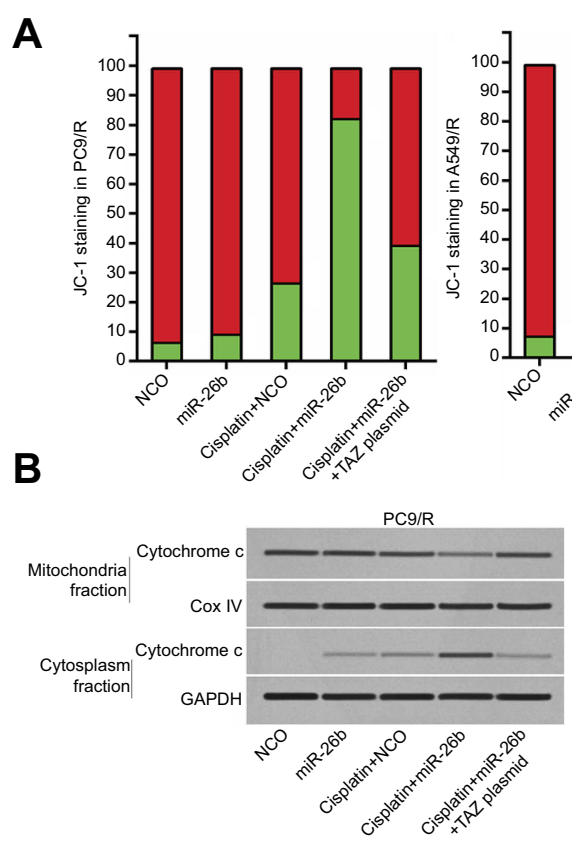
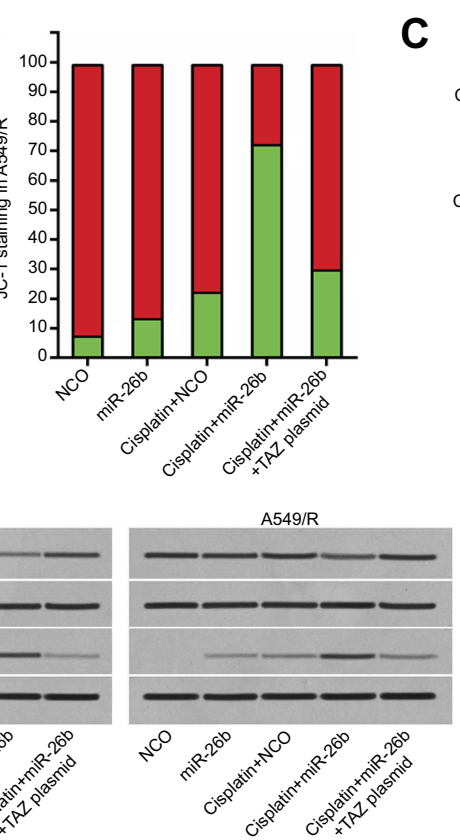

C
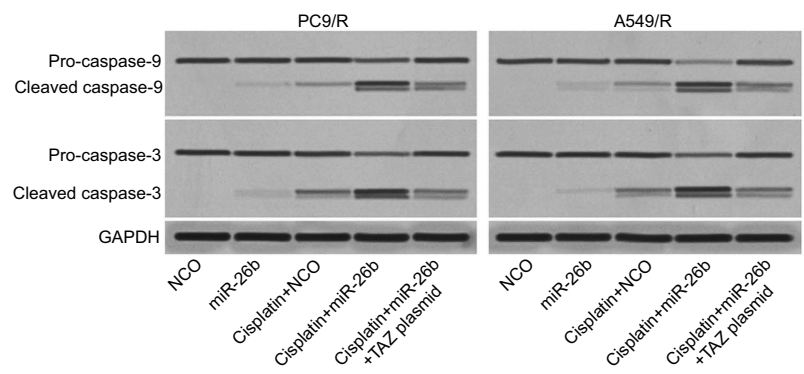

D

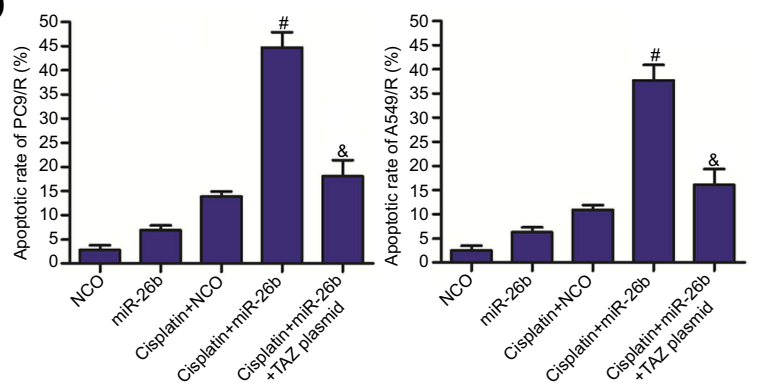

Figure 5 MiR-26b mimics targets TAZ to enhance the cisplatin-dependent mitochondrial apoptosis in A549/R and PC9/R. (A) Transfection with miR-26b mimics promoted the collapse of MMP in A549/R and PC9/R cells which were under the cisplatin (I5 $\mu \mathrm{M})$ treatment. (B) Transfection with miR-26b mimics promoted the release of cytochrome c from mitochondria into cytosol of A549/R and PC9/R cells which were under the cisplatin (I5 $\mu \mathrm{M}$ ) treatment. (C) Transfection with miR-26b mimics promoted the cleavage of caspase-9 and caspase-3 in A549/R and PC9/R cells which were under the cisplatin (I5 $\mu \mathrm{M})$ treatment. (D) Transfection with miR-26b mimics increased the apoptotic rate of PC9/R and A549/R cells which were under the cisplatin (I5 $\mu \mathrm{M})$ treatment.

Notes: Data are expressed as mean \pm SD. ${ }^{\#} P<0.05$ vs Cisplatin+NCO group, ${ }^{\&} P<0.05$ vs Cisplatin+miR-26b group.

Abbreviations: TAZ, Tafazzin; MMP, mitochondrial membrane potential; NSCLC, non-small cell lung cancer; NCO, negative control oligonucleotide.

\section{Restoration of miR-26b expression decreases the cross-resistance of PC9/R and $A 549 / R$ to other platinum-based chemotherapeutic drugs}

Results of CCK-8 assays showed that cisplatin-resistant PC9 and A549 cells exhibited significant cross-resistance to carboplatin (Figure 6A) and oxaliplatin (Figure 6B). To investigate whether the cross-resistance of PC9/R and A549/R to carboplatin and oxaliplatin was dependent on the absence of miR-26b, we transfected the PC9/R and A549/R cells with miR-26b mimics before the evaluation of IC50 to carboplatin and oxaliplatin. We then found that restoration of miR-26b in PC9/R and A549/R cells obviously decreased the IC50 of carboplatin (Figure 6C) and oxaliplatin (Figure 6D) to them. We demonstrated that overexpression of miR-26b was able to reverse the cross-resistance of NSCLC cells to platinumbased drugs.

\section{Discussion}

Formation of acquired drug resistance is still a major obstacle during the course of cisplatin-based chemotherapy for NSCLC patients. Novel strategies are urgent to be explored to reverse or impede the cisplatin resistance in NSCLC treatment. Recent studies have demonstrated that formation of cisplatin resistance is partially induced by dysregulation of miRNAs in cancers including NSCLC. ${ }^{24-26}$ MiRNAs have been considered as potential targets for improving the cisplatin-based treatment. Among these cancer-related miRNAs, miR-26b acts as a tumor suppressor and is always downregulated in multiple cancers. ${ }^{27,28}$ Furthermore, previous studies have found that the absence of miR-26b is associated with chemoresistance in cancers, whereas overexpression of miR-26b sensitizes these cancer cells to chemotherapeutic drugs including cisplatin. ${ }^{29-31}$ In the present study, we demonstrated that miR-26b expression was downregulated in the established cisplatin-resistant NSCLC models. Furthermore, the reduction of miR-26b was associated with the formation of cisplatin resistance in these cisplatin-resistant NSCLC models. Restoration of miR-26b was found to partially reverse the cisplatin resistance.

TAZ is a mitochondria-related protein that locates on the mitochondrial membrane. TAZ functions to remodel the cardiolipin that is a major lipid in the mitochondrial membrane. Thus, TAZ is important to keep the 

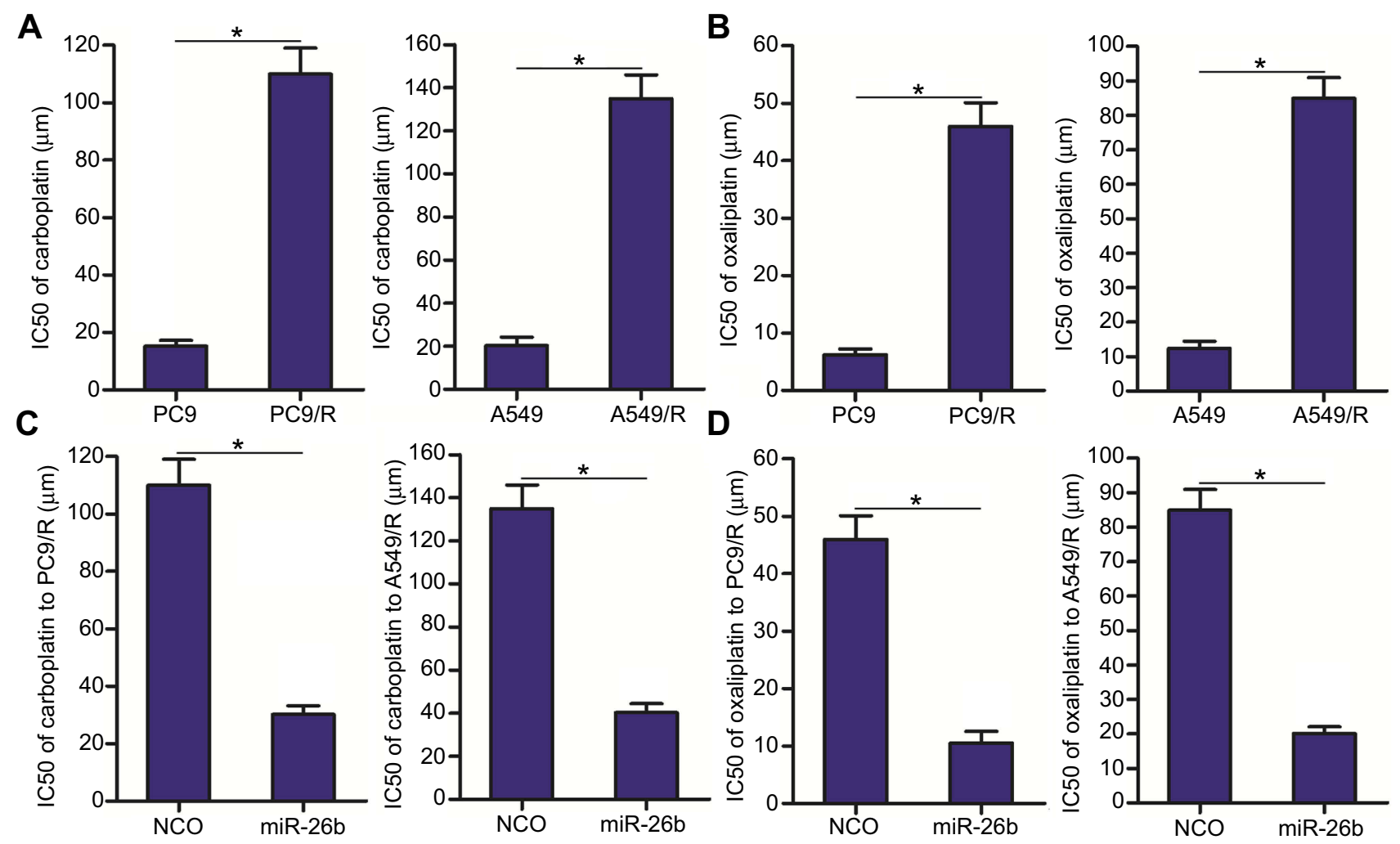

Figure 6 MiR-26b mimics reversed the cross-resistance of A549/R and PC9/R to carboplatin and oxaliplatin. (A) Cross-resistance of A549/R and PC9/R to carboplatin. (B) Cross-resistance of A549/R and PC9/R to oxaliplatin. (C) Transfection with miR-26b mimics decreased the IC50 of A549/R and PC9/R to carboplatin. (D) Transfection with miR-26b mimics decreased the IC50 of A549/R and PC9/R to oxaliplatin.

Notes: Data are expressed as mean \pm SD. $* P<0.05$.

Abbreviations: IC50, 50\% inhibiting concentration; NCO, negative control oligonucleotide.

stabilization of mitochondrial outer membrane. ${ }^{19,20}$ Previous studies have reported that TAZ is overexpressed in multiple cancers. ${ }^{32,33}$ Furthermore, research has indicated that TAZ promotes drug resistance in some cancers. Inhibition of TAZ can improve the chemosensitivity of some cancer cells. ${ }^{34,35}$ These studies suggest that suppression of TAZ expression may be a potential strategy for impeding the drug resistance.

In our study, we proved that the resistance of cisplatin in established cisplatin-resistant NSCLC models was partially induced by the overexpression of TAZ. Furthermore, we demonstrated that overexpression of TAZ was induced by the absence of miR-26b in cisplatin-resistant NSCLC cells. Our data proved that restoration of miR-26b expression can partially reverse the cisplatin resistance of NSCLC through suppression of TAZ.

As cisplatin damages cellular DNA, cisplatin induces mitochondrial apoptosis of cancer cells. ${ }^{36,37}$ TAZ is involved in maintaining the stability of mitochondrial outer membrane to keep the MMP. ${ }^{38}$ Thus, reduction of TAZ expression can decrease the stability of mitochondrial outer membrane to facilitate the mitochondrial pathway of apoptosis. In our research to explore the mechanisms by which miR-26b partially reversed the cisplatin resistance of NSCLC, we demonstrated that cisplatin damaged DNA of cisplatin-resistant NSCLC cells to provide the apoptosis signals. Meanwhile, miR-26b inhibited TAZ expression and thus reduced the stability of mitochondrial outer membrane. As the results, miR-26b facilitated the mitochondria collapse in cancer cells which were under the stress of cisplatin-provided apoptosis signals. Due to the mitochondria collapse, MMP was decreased and the mitochondrial outer membrane permeability was induced. Subsequently, cytochrome c was released from the mitochondria into the cytosol followed by activation of effector caspases to cause the apoptosis of cisplatin-resistant NSCLC cells (Figure 7).

Taken together, these results highlight that restoration of miR-26b expression can partially reverses the cisplatin resistance of NSCLC. Decreasing of TAZ by a miR-26b-based 


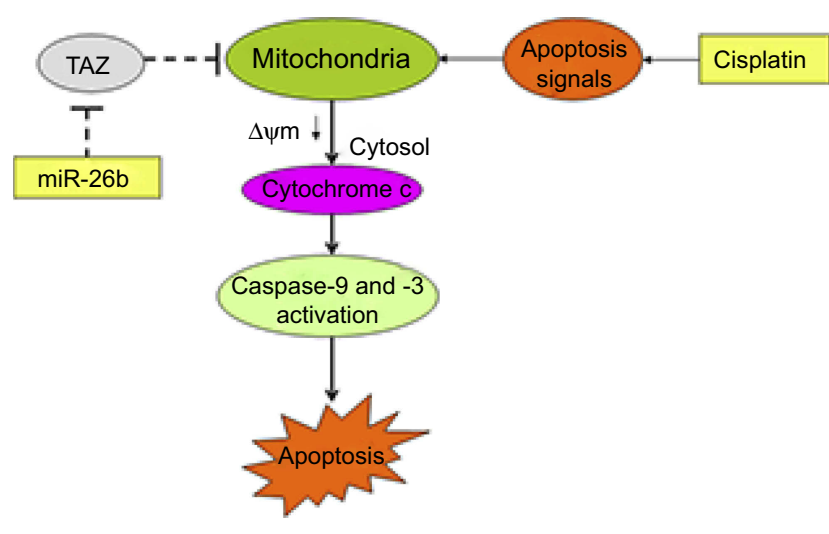

Figure 7 Schema of the predicted mechanisms by which miR-26b partially reverses the cisplatin resistance of NSCLC. Cisplatin damages DNA of A549/R and PC9/R cells to provide the apoptosis signals. Meanwhile, miR-26b inhibits TAZ expression and thus reduces the stability of mitochondrial outer membrane. As the results, miR-26b facilitates the mitochondria collapse in cancer cells which are under the stress of cisplatin-provided apoptosis signals. Due to the mitochondria collapse, $\Delta \varphi$ is decreased and the MOM permeability is induced. Subsequently, cytochrome $c$ is released from the mitochondria into the cytosol followed by activation of effector caspases to cause the apoptosis of A549/R and PC9/R cells.

Abbreviations: TAZ, Tafazzin; NSCLC, non-small cell lung cancer; $\Delta \varphi$, mitochondrial membrane potential; MOM, mitochondrial outer membrane.

approach may represent a potential strategy to reverse the cisplatin resistance in NSCLC.

\section{Acknowledgment}

We thank all the contributors who assisted us during this study.

\section{Disclosure}

The authors report no conflicts of interest in this work.

\section{References}

1. Siegel RL, Miller KD, Jemal A. Cancer statistics, 2019. CA Cancer J Clin. 2019;69:7-34. doi:10.3322/caac.21551

2. Wen YS, Xi KX, Xi KX, et al. The number of resected lymph nodes is associated with the long-term survival outcome in patients with T2 N0 non-small cell lung cancer. Cancer Manag Res. 2018;10:6869-6877. doi: $10.2147 / C M A R . S 186047$

3. Tong KM, Laskin J, Ho C. Maintenance chemotherapy in advanced NSCLC: a population-based assessment of eligibility. Lung Cancer. 2015;87:296-302. doi:10.1016/j.lungcan.2014.12.014

4. Wang S, Fu Y, Ma K, et al. The significant increase and dynamic changes of the myeloid-derived suppressor cells percentage with chemotherapy in advanced NSCLC patients. Clin Transl Oncol. 2014;16:616-622. doi:10.1007/s12094-013-1125-y

5. Wen-Xiu X, Xiao-Wei Z, Hai-Ying D, et al. Impact of metformin use on survival outcomes in non-small cell lung cancer treated with platinum. Medicine (Baltimore). 2018;97:e13652. doi:10.1097/MD.0000000 000013652

6. Li YQ, Chen J, Yin JY, et al. Gene expression and single nucleotide polymorphism of ATP7B are associated with platinum-based chemotherapy response in non-small cell lung cancer patients. $J$ Cancer. 2018;9:3532-3539. doi:10.7150/jca.26286

7. Inoue A, Kikuchi S, Hishiki A, et al. A small molecule inhibitor of monoubiquitinated proliferating cell nuclear antigen (PCNA) inhibits repair of interstrand DNA cross-link, enhances DNA double strand break, and sensitizes cancer cells to cisplatin. J Biol Chem. 2014;289:7109-7120. doi:10.1074/jbc.M113.520429
8. Ming X, Groehler A 4th, Michaelson-Richie ED, et al. Mass spectrometry based proteomics study of cisplatin-induced DNAprotein cross-linking in human fibrosarcoma (HT1080) cells. Chem Res Toxicol. 2017;30:980-995. doi:10.1021/acs.chemrestox.6b 00389

9. Gao BB, Wang SX. LncRNA BC200 regulates the cell proliferation and cisplatin resistance in non-small cell lung cancer via PI3K/AKT pathway. Eur Rev Med Pharmacol Sci. 2019;23:1093-1101. doi:10.26355/eurrev_201902_16999

10. $\mathrm{Li} \mathrm{Y}, \mathrm{He} \mathrm{LR}, \mathrm{Gao} \mathrm{Y}$, et al. CHD1L contributes to cisplatin resistance by upregulating the ABCB1-NF- $\mathrm{KB}$ axis in human non-small-cell lung cancer. Cell Death Dis. 2019;10:99. doi:10.1038/s41419-019-1371-1

11. Bartel DP. MicroRNAs: genomics, biogenesis, mechanism, and function. Cell. 2004;116:281-297. doi:10.1016/s0092-8674(04)00045-5

12. Farazi TA, Hoell JI, Morozov P, et al. MicroRNAs in human cancer. Adv Exp Med Biol. 2013;774:1-20. doi:10.1007/978-94-007-5590-1_1

13. Lee YS, Dutta A. MicroRNAs in cancer. Annu Rev Pathol. 2009;4:199-227. doi:10.1146/annurev.pathol.4.110807.092222

14. Ganju A, Khan S, Hafeez BB, et al. miRNA nanotherapeutics for cancer. Drug Discov Today. 2017;22:424 432. doi:10.1016/j.drudis.2016.10.014

15. Tutar L, Özgür A, Tutar Y. Involvement of miRNAs and pseudogenes in cancer. Methods Mol Biol. 2018;1699:45-66. doi:10.1007/978-14939-7435-1_3

16. Tormo E, Ballester S, Adam-Artigues A, et al. The miRNA-449 family mediates doxorubicin resistance in triple-negative breast cancer by regulating cell cycle factors. Sci Rep. 2019;9:5316. doi:10.1038/s41598-019-41472-y

17. Zhang Y, He Y, Lu LL, et al. miRNA-192-5p impacts the sensitivity of breast cancer cells to doxorubicin via targeting peptidylprolyl isomerase A. Kaohsiung J Med Sci. 2019;35:17-23. doi:10.1002/ kjm2.12004

18. Xie X, Huang N, Zhang Y, et al. MiR-192-5p reverses cisplatin resistance by targeting ERCC3 and ERCC4 in SGC7901/DDP cells. J Cancer. 2019;10:1039-1051. doi:10.7150/jca.25814

19. Houtkooper RH, Turkenburg M, Poll-The BT, et al. The enigmatic role of tafazzin in cardiolipin metabolism. Biochim Biophys Acta. 2009;1788:2003-2014. doi:10.1038/srep42748

20. Chen M, Zhang Y, Zheng PS. Tafazzin (TAZ) promotes the tumorigenicity of cervical cancer cells and inhibits apoptosis. PLoS One. 2017;12:e177171. doi:10.1371/journal.pone.0177171

21. Zhang R, Xu J, Zhao J, et al. Knockdown of miR-27a sensitizes colorectal cancer stem cells to TRAIL by promoting the formation of Apaf-1-caspase-9 complex. Oncotarget. 2017;8:45213-45223. doi:10.18632/oncotarget.16779

22. Sun Y, He N, Dong Y, et al. MiR-24-BIM-Smac/DIABLO axis controls the sensitivity to doxorubicin treatment in osteosarcoma. Sci Rep. 2016;6:34238. doi:10.1038/srep34238

23. Wang R, Wen B, Sun D. miR-573 regulates cell proliferation and apoptosis by targeting Bax in nucleus pulposus cells. Cell Mol Biol Lett. 2019;24:2. doi:10.1186/s11658-018-0132-y

24. Zhang L, He L, Zhang H, et al. Knockdown of miR-20a enhances sensitivity of colorectal cancer cells to cisplatin by increasing ASK1 expression. Cell Physiol Biochem. 2018;47:1432-1441. doi:10.1159/ 000490834

25. Zhou S, Huang Q, Zheng S, et al. miR-27a regulates the sensitivity of breast cancer cells to cisplatin treatment via BAK-SMAC/DIABLOXIAP axis. Tumour Biol. 2016;37:6837-6845. doi:10.1007/s13277015-4500-1

26. Sun W, Ping W, Tian Y, et al. miR-202 enhances the anti-tumor effect of cisplatin on non-small cell lung cancer by targeting the Ras/MAPK pathway. Cell Physiol Biochem. 2018;51:2160-2171. doi:10.1159/ 000495835

27. Li J, Li X, Kong X, et al. MiRNA-26b inhibits cellular proliferation by targeting CDK8 in breast cancer. Int J Clin Exp Med. 2014;7:558-565. 
28. Shen G, Lin Y, Yang X, et al. MicroRNA-26b inhibits epithelialmesenchymal transition in hepatocellular carcinoma by targeting USP9X. BMC Cancer. 2014;14:393. doi:10.1186/1471-2407-14-393

29. Shi L, Yin W, Zhang Z, et al. Down-regulation of miR-26b induces cisplatin resistance in nasopharyngeal carcinoma by repressing JAG1. FEBS Open Bio. 2016;6:1211-1219. doi:10.1002/2211-5463.12135

30. Jin F, Wang Y, Li M, et al. MiR-26 enhances chemosensitivity and promotes apoptosis of hepatocellular carcinoma cells through inhibiting autophagy. Cell Death Dis. 2017;8:e2540. doi:10.1038/ cddis. 2016.461

31. Liang N, Zhou X, Zhao M, et al. Down-regulation of microRNA-26b modulates non-small cell lung cancer cells chemoresistance and migration through the association of PTEN. Acta Biochim Biophys Sin (Shanghai). 2015;47:530-538. doi:10.1093/abbs/gmv046

32. Pathak S, Meng WJ, Zhang H, et al. Tafazzin protein expression is associated with tumorigenesis and radiation response in rectal cancer: a study of Swedish clinical trial on preoperative radiotherapy. PLoS One. 2014;9:e98317. doi:10.1371/journal.pone.0098317

33. Chevillard S, Ugolin N, Vielh P, et al. Gene expression profiling of differentiated thyroid neoplasms: diagnostic and clinical implications. Clin Cancer Res. 2004;10:6586-6597. doi:10.1158/1078-0432.CCR04-0053
34. Liu J, Li J, Li P, et al. DLG5 suppresses breast cancer stem cell-like characteristics to restore tamoxifen sensitivity by inhibiting TAZ expression. J Cell Mol Med. 2019;23:512-521. Epub 2018 Nov 18.

35. Xu W, Wei Y, Li Y, et al. TAZ inhibition restores sensitivity of cisplatin via AKT/mTOR signaling in lung adenocarcinoma. Oncol Rep. 2017;38:1815-1821. doi:10.3892/or.2017.5847

36. Lee SI, Jeong YJ, Yu AR, et al. Carfilzomib enhances cisplatininduced apoptosis in SK-N-BE(2)-M17 human neuroblastoma cells. Sci Rep. 2019;9:5039. doi:10.1038/s41598-019-41527-0

37. Tusskorn O, Khunluck T, Prawan A, et al. Mitochondrial division inhibitor-1 potentiates cisplatin-induced apoptosis via the mitochondrial death pathway in cholangiocarcinoma cells. Biomed Pharmacother. 2019;111:109-118. doi:10.1016/j.biopha.2018.12.051

38. Gonzalvez F, D'Aurelio M, Boutant M, et al. Barth syndrome: cellular compensation of mitochondrial dysfunction and apoptosis inhibition due to changes in cardiolipin remodeling linked to tafazzin (TAZ) gene mutation. Biochim Biophys Acta. 2013;1832:1194-1206. doi:10.1016/j.bbadis.2013.03.005

\section{Publish your work in this journal}

OncoTargets and Therapy is an international, peer-reviewed, open access journal focusing on the pathological basis of all cancers, potential targets for therapy and treatment protocols employed to improve the management of cancer patients. The journal also focuses on the impact of management programs and new therapeutic agents and protocols on patient perspectives such as quality of life, adherence and satisfaction. The manuscript management system is completely online and includes a very quick and fair peer-review system, which is all easy to use. Visit http://www.dovepress.com/ testimonials.php to read real quotes from published authors. 